\title{
OSCILLATORS NEAR HOPF BIFURCATION
}

In this paper the differential transformation method (DTM) is employed to solve a system of linear differential equations derived for energy optimal control theory and nonlinear differential equations and their systems for oscillators near Hopf bifurcation. They are given by differential equations in a complex plane. Different types of forced terms are considered. The approximate solutions are given in the form of series with required accuracy. Some numerical examples are provided.

Keywords: Oscillators, systems of ordinary differential equations, differential transformation method.

\section{Introduction}

The collective behaviour of oscillators and coupled oscillators represents the complex dynamic system. It demonstrates interest in experimental as well as numerical research. Examples of such applications may be found in mechanical oscillators, limit cycle oscillators, semiconductor lasers, superconducting Josephson junction or pacemakers. Synchronization, where individual oscillators oscillate at a common phase and frequency, depends on coupling force. A weak coupling leads to the character of non-coupled oscillators, for stronger coupling the considering of both frequency and amplitude is required. An approach used in the investigation is based on the study of the stability - the system is linearized near zero [1]. The solving of differential equations or their systems by DTM avoids such a restriction. This paper presents two main goals. Firstly, we apply the differential transformation method for oscillators and coupled oscillators. Secondly, we reduce a system of linear equations in an engineering application to the system of linear equations to energy optimal control of motors.

\section{DTM - differential transformation method}

In this section we bring a brief overview of the differential transformation method that proposes a technique which does not evaluate the derivatives symbolically; the differential transformation DT of the $n^{\text {th }}$ derivative of a function $y(t)$ is defined by
$Y(n)=\frac{1}{n !}\left[\frac{d^{n} y(t)}{d t^{n}}\right]_{t=t_{0}}$

where $y(t)$ is an original and $Y(t)$ is a transformed function.

An inverse transformation of $\mathrm{Y}(\mathrm{n})$ leads to

$y(t)=\sum_{n=0}^{\infty} Y(n)\left(t-t_{0}\right)^{n}$.

If $t_{0}=0$, then the representation of the function reduces to

$y(t)=\sum_{n=0}^{\infty} Y(n) t^{n}$

The properties of the DT with their proofs are published in [2 - 4]; the used ones of them are introduced below.

Let functions $G, G_{i}, i=1,2,3$ be the differential transformations of functions $g, g_{i}, i=1,2,3$, then the following correspondences hold

$g(t)=g_{1}(t)+g_{2}(t) \rightarrow G(n)=G_{1}(n)+G_{2}(n)$

$g(t)=\lambda g_{1}(t) \rightarrow G(n)=\lambda G_{1}(n)$

$g(t)=g_{1}(t) g_{2}(t) \rightarrow G(n)=\sum_{l=0}^{n} G_{1}(l) G_{2}(n-l)(6)$

$g(t)=t^{k} \rightarrow G(n)=\delta(n-k)$

$g(t)=e^{\beta t} \rightarrow G(n)=\frac{\beta^{n}}{n !}$

$g(t)=\frac{d^{k} g_{1}(t)}{d t^{k}} \rightarrow G(n)=\frac{(n+k) !}{n !} G_{1}(n+k)$

\footnotetext{
* ${ }^{1}$ Helena Samajova, ${ }^{2}$ Tongxing Li

${ }^{1}$ Department of Appl. Mathematics, Faculty of Mechanical Engineering, University of Zilina, Slovakia

${ }^{2}$ School of Control Science and Engineering, Shandong University, Jinan, China

E-mail: helena.samajova@fstroj.uniza.sk
} 


$$
\begin{aligned}
& g(t)=\prod_{i=1}^{3} g_{i}(t) \rightarrow G(n)=\sum_{s_{1}=0}^{n} \sum_{s_{2}=0}^{n-s_{1}} G_{1}\left(s_{1}\right) G_{2}\left(s_{2}\right) . \\
& G_{3}\left(n-s_{1}-s_{2}\right)
\end{aligned}
$$

where $\delta(n-k)$ is the Kronecker delta symbol, $n, l, k \in N^{0}$.

\section{Oscillators near Hopf bifurcation}

\subsection{Differential equation for an oscillator with a real parameter $\omega$.}

We consider nonlinear differential equation used for oscillators [1] near Hopf bifurcation [5] with different types of forcing terms

$y^{\prime}(t)=y(t)\left[1+j \omega+|y(t)|^{2}\right]+f(t)$,

where $j^{2}=-1, \omega \in \mathbf{R}$, with the initial condition $y(0)=a$ and $a \in C$ is a constant. With the use of $y(t)=u(t)+j v(t)$ and a forcing term $f(t)=f_{1}(t)+j f_{2}(t)$ the equation (11) is equivalent to the system of two equations

$u^{\prime}(t)=u(t)-\omega v(t)+u^{3}(t)+u(t) v^{2}(t)+f_{1}(t)$

$v^{\prime}(t)=v(t)+\omega u(t)+v(t) u^{2}(t)+v^{3}(t)+f_{2}(t)$

Using the properties (4) - (10), the resulting system of the DTM is

$$
\begin{aligned}
& U(n+1)=\left[U(n)-\omega V(n)+\sum_{s_{1}=0}^{n} \sum_{s_{2}=0}^{n-s_{1}} U\left(s_{1}\right) U\left(s_{2}\right) .\right. \\
& \cdot U\left(n-s_{1}-s_{2}\right)+\sum_{s_{1}=0}^{n} \sum_{s_{2}=0}^{n-s_{1}} U\left(s_{1}\right) V\left(s_{2}\right) . \\
& \left.\cdot V\left(n-s_{1}-s_{2}\right)+F_{1}(n)\right] /(n+1)
\end{aligned}
$$$$
V(n+1)=\left[V(n)+\omega U(n)+\sum_{s_{1}=0}^{n} \sum_{s_{2}=0}^{n-s_{1}} V\left(s_{1}\right) U\left(s_{2}\right) .\right.
$$$$
U\left(n-s_{1}-s_{2}\right)+\sum_{s_{1}=0}^{n} \sum_{s_{2}=0}^{n-s_{1}} V\left(s_{1}\right) V\left(s_{2}\right) \text {. }
$$$$
\left.\cdot V\left(n-s_{1}-s_{2}\right)+F_{2}(n)\right] /(n+1)
$$

Now we will consider the cases with forcing terms of different types.

R1.Consider equations (12) without any forcing term and with the initial conditions $u(0)=0.5, v(0)=0.75$ and a parameter $\omega=2$. The transformation of functions $f_{1}(t)=f_{2}(t)=0$ yields $F_{1}(n)=F_{2}(n)=0$.

The Matlab program is involved and gives numerical solutions as follows

$u(t)=0.5-0.3906 t-0.4091 t^{2}+2.9799 t^{3}+6.6069 t^{4}+8.7719 t^{5}+$.

$v(t)=0.75+2.1562 t+1.2397 t^{2}+0.7014 t^{3}+2.5814 t^{4}+5.9462 t^{5}+\ldots$
$\mathrm{R} 2 \mathrm{a})$. For the forcing term of the power type $f(t)=t^{k}+j t^{l}$, where $k, l$ are nonnegative integers the DT of $f_{1}(t)$ and $f_{2}(t)$ are $F_{1}(n)=\delta(n-k), F_{2}(n)=\delta(n-l)$. The solutions for the same conditions as in R1. and $k=3, l=4$ are

$u(t)=0.5-0.3906 t-0.4091 t^{2}+2.9799 t^{3}+6.8569 t^{4}+8.8594 t^{5}+\ldots$ $v(t)=0.75+2.1562 t+1.2397 t^{2}+0.7014 t^{3}+2.3814 t^{4}+6.311 t^{5}+\ldots$

b) Solutions under the initial conditions $u(0)=0.5$ $v(0)=0.75$ and parameters $\omega=2$ and $k=3$ are given as $u(t)=0.5-0.3906 t-0.4091 t^{2}+2.9799 t^{3}+6.8569 t^{4}+8.8563 t^{5}+\ldots$ $v(t)=0.75+2.1562 t+1.2397 t^{2}+0.7014 t^{3}+2.3814 t^{4}+6.19935 t^{5}+\ldots$

R3. If the forcingtermis of the exponential type $f(t)=e^{\alpha t}+j e^{\beta t}$, the DT of $f(t)$ is $F(n)=F_{1}(n)+j F_{2}(n)$,

where $F_{1}(n)=\frac{\alpha^{n}}{n !}, F_{2}(n) \frac{\beta^{n}}{n !}$.

For $\alpha=2, \beta=1$ and for identical initial condition we obtain following solutions

$u(t)=0.5-0.6093 t-1.4345 t^{2}+9.6199 t^{3}+29.8612 t^{4}+75.7508 t^{5}+\ldots$ $v(t)=0.75+3.1562 t+4.2701 t^{2}+7.0497 t^{3}+19.3663 t^{4}+55.678 t^{5}+\ldots$

In the special case $\alpha=\beta=1$

$u(t)=0.5-0.6093 t-0.9345 t^{2}+8.8282 t^{3}+28.7231 t^{4}+71.9794 t^{5}+\ldots$ $v(t)=0.75+3.1562 t+4.271 t^{2}+6.4976 t^{3}+17.6488 t^{4}+50.7307 t^{5}+\ldots$

Remark: From the theory of Taylor series and relations (7), (8) and (13), it is evident that the influence of the power type right hand side (RHS) manifests from the term of the lower degree of $k, l$ unlike the RHS of exponential type changes all the terms of solutions.

\subsection{Differential equation for an oscillator with a complex parameter $\omega$.}

Consider the equation (11) with $\omega=\omega_{1}+j \omega_{2} \in C$. The equation is separated into

$u^{\prime}(t)=u(t)\left(1+\omega_{1}\right)-\omega_{2} v(t)+u^{3}(t)+$

$+u(t) v^{2}(t)+f_{1}(t)$

$v^{\prime}(t)=v(t)\left(1+\omega_{2}\right)+\omega_{1} u(t)+$

$+v(t) u^{2}(t)+v^{3}(t)+f_{2}(t)$ 

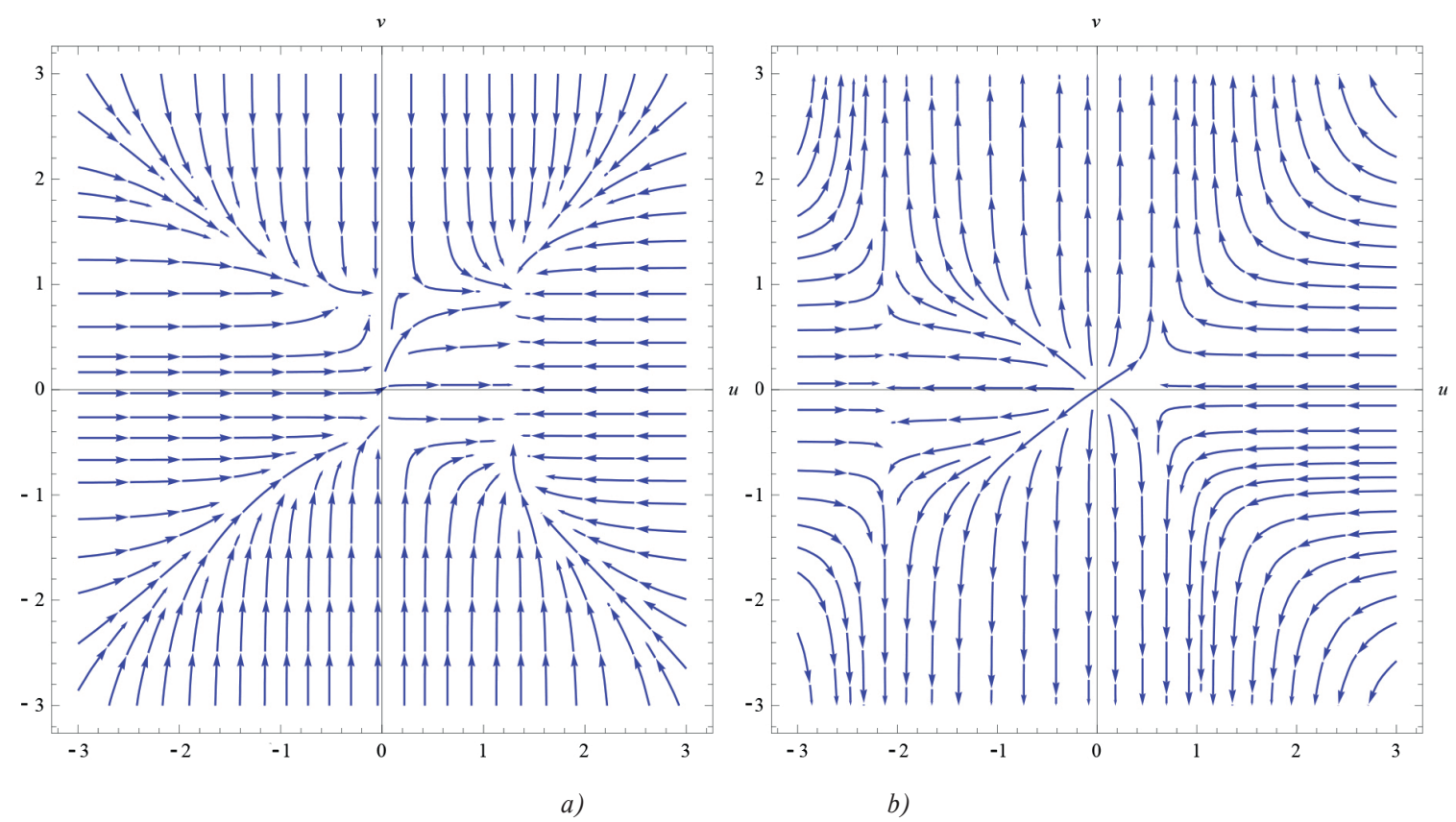

Fig. 1 Vector fields

Such kind of a system could be regarded as coupled oscillators with two different frequencies $\omega_{1}, \omega_{2}$.

The DM of $u(t), v(t)$

$$
\begin{aligned}
& U(n+1)=\left[\left(1+\omega_{1}\right) U(n)-\omega_{2} V(n)+\sum_{s_{1}=0}^{n} \sum_{s_{2}=0}^{n-s_{1}} U\left(s_{1}\right) .\right. \\
& \cdot U\left(s_{2}\right) U\left(n-s_{1}-s_{2}\right)+\sum_{s_{1}=0}^{n} \sum_{s_{2}=0}^{n-s_{1}} U\left(s_{1}\right) V\left(s_{2}\right) . \\
& \left.\cdot V\left(n-s_{1}-s_{2}\right)+F_{1}(n)\right] /(n+1)
\end{aligned}
$$

$$
\begin{aligned}
& V(n+1)=\left[\left(1+\omega_{2}\right) V(n)+\omega_{1} U(n)+\sum_{s_{1}=0}^{n} \sum_{s_{2}=0}^{n-s_{1}} V\left(s_{1}\right) .\right. \\
& \cdot U\left(s_{2}\right) U\left(n-s_{1}-s_{2}\right)+\sum_{s_{1}=0}^{n} \sum_{s_{2}=0}^{n-s_{1}} V\left(s_{1}\right) V\left(s_{2}\right) . \\
& \left.\cdot V\left(n-s_{1}-s_{2}\right)+F_{2}(n)\right] /(n+1)
\end{aligned}
$$

The difference of two frequencies $\Delta=\left|\omega_{1}-\omega_{2}\right|$ and a coupling constant $K$ introduced in the system of differential equations (23) have a decisive influence on the behaviour of such systems of coupled oscillators. It could be one of the parameters which may be the reason of quenching of oscillators' amplitudes.

The numerical solutions of (21) are given for the initial conditions $u(0)=0.5, v(0)=0.75$ and frequencies $\omega_{1}=1, \omega_{2}=2$

$u(t)=0.5-0.1563 t-0.1143 t^{2}+0.7296 t^{3}+2.4144 t^{4}+6.4313 t^{5}+\ldots$ $v(t)=0.75+2.3594 t+3.6802 t^{2}+7.8027 t^{3}+19.3675 t^{4}+48.2082 t^{5}+\ldots$
Vector fields for solutions of equation (19) near Hopf bifurcation with initial conditions $u(0)=v(0)=0.001$, $\omega=5+6 j \mathrm{n}=20$ are in Fig. 1 with forcing term

a) $f(t)=t-j t^{4}$; b) $f(t)=e+j e^{-3}$.

\subsection{System of differential equations for coupled oscillators with real parameters $\omega_{1}, \omega_{2}$.}

Now, let us consider the system of two coupled oscillators with a constant $K$, which represents the coupling as considered in [1] and $\omega_{1}, \omega_{2} \in \mathbf{R}$

$$
\begin{aligned}
& y_{1}^{\prime}(t)=y_{1}(t)\left[1+j \omega_{1}+\left|y_{1}(t)\right|^{2}\right]+K\left(y_{2}(t)-y_{1}(t)\right) \\
& y_{2}^{\prime}(t)=y_{2}(t)\left[1+j \omega_{2}+\left|y_{2}(t)\right|^{2}\right]+K\left(y_{1}(t)-y 2(t)\right)
\end{aligned}
$$

Similarly to the case of one equation we may separate it into the system

$u_{1}^{\prime}(t)=u_{1}(t)-\omega_{1} v_{1}(t)+u_{1}^{3}(t)+$

$+u_{1}(t) v_{1}^{2}(t)+K\left(u_{2}(t)-u_{1}(t)\right)$

$v_{1}^{\prime}(t)=v_{1}(t)+\omega_{1} u_{1}(t)+u_{1}^{2}(t) v_{1}(t)+$

$+v_{1}^{3}(t)+K\left(v_{2}(t)-v_{1}(t)\right)$

$u_{2}^{\prime}(t)=u_{2}(t)-\omega_{2} v_{2}(t)+u_{2}^{3}(t)+$

$+u_{2}(t) v_{2}^{2}(t)+K\left(u_{1}(t)-u_{2}(t)\right)$

$v_{2}^{\prime}(t)=v_{2}(t)+\omega_{2} u_{2}(t)+u_{2}^{2}(t) v_{2}(t)+$

$+v_{2}^{3}(t)+K\left(v_{1}(t)-v_{2}(t)\right)$ 
and the DT is

$$
\begin{aligned}
& U_{1}(n+1)=\left[U_{1}(n)-\omega_{1} V_{1}(n)+\sum_{s_{1}=0}^{n} \sum_{s_{2}=0}^{n-s_{1}} U_{1}\left(s_{1}\right) .\right. \\
& U_{1}\left(s_{2}\right) U_{1}\left(n-s_{1}-s_{2}\right)+\sum_{s_{1}=0}^{n} \sum_{s_{2}=0}^{n-s_{1}} U_{1}\left(s_{1}\right) V_{1}\left(s_{2}\right) . \\
& \left.\cdot V_{1}\left(n-s_{1}-s_{2}\right)+K\left(U_{2}(n)-U_{1}(n)\right)\right] /(n+1) \\
& V_{1}(n+1)=\left[V_{1}(n)+\omega_{1} U_{1}(n)+\sum_{s_{1}=0}^{n} \sum_{s_{2}=0}^{n-s_{1}} V_{1}\left(s_{1}\right) .\right. \\
& U_{1}\left(s_{2}\right) U_{1}\left(n-s_{1}-s_{2}\right)+\sum_{s_{1}=0}^{n} \sum_{s_{2}=0}^{n-s_{1}} V_{1}\left(s_{1}\right) V_{1}\left(s_{2}\right) . \\
& \left.\cdot V_{1}\left(n-s_{1}-s_{2}\right)+K\left(V_{2}(n)-V_{1}(n)\right)\right] /(n+1)
\end{aligned}
$$$$
U_{2}(n+1)=\left[U_{2}(n)-\omega_{2} V_{2}(n)+\sum_{s_{1}=0}^{n} \sum_{s_{2}=0}^{n-s_{1}} U_{2}\left(s_{1}\right) .\right.
$$$$
U_{2}\left(s_{2}\right) U_{2}\left(n-s_{1}-s_{2}\right)+\sum_{s_{1}=0}^{n} \sum_{s_{2}=0}^{n-s_{1}} U_{2}\left(s_{1}\right) V_{2}\left(s_{2}\right) .
$$$$
\left.\cdot V_{2}\left(n-s_{1}-s_{2}\right)+K\left(U_{1}(n)-U_{2}(n)\right)\right] /(n+1)
$$$$
V_{2}(n+1)=\left[V_{2}(n)+\omega_{2} U_{2}(n)+\sum_{s_{1}=0}^{n} \sum_{s_{2}=0}^{n-s_{1}} V_{2}\left(s_{1}\right)\right.
$$$$
U_{2}\left(s_{2}\right) U_{2}\left(n-s_{1}-s_{2}\right)+\sum_{s_{1}=0}^{n} \sum_{s_{2}=0}^{n-s_{1}} V_{2}\left(s_{1}\right) V_{2}\left(s_{2}\right) .
$$$$
\left.\cdot V_{2}\left(n-s_{1}-s_{2}\right)+K\left(V_{1}(n)-V_{2}(n)\right)\right] /(n+1)
$$

Considering the parameters of previous numerical examples $u_{1}(0)=u_{2}(0)=0.5, v_{1}(0)=v_{2}(0)=0.75, \omega_{1}=2$, $\omega_{2}=1, K=2$, the components of the solutions are as follows

$u_{1}(t)=0.5-0.5938 t-1.4111 t^{2}-1.1733 t^{3}-2.7805 t^{4}-4.5852 t^{5}+\ldots$ $v_{1}(t)=0.75+2.3594 t+2.1489 t^{2}+5.4193 t^{3}+11.1783 t^{4}+25.7515 t^{5}+\ldots \quad(25)$ $u_{2}(t)=0.5+0.1563 t-0.8018 t^{2}-0.5712 t^{3}+0.3199 t^{4}+0.2545 t^{5}+\ldots$ $v_{2}(t)=0.75+1.8594 t+3.3677 t^{2}+4.7132 t^{3}+11.9392 t^{4}+26.1863 t^{5}+\ldots$

\section{An engineering application}

Let us demonstrate the exploitation of DTM principles on the problem describing an electric motor presented in [6 and 7]. Mathematical analysis of energy optimal speed and position control of the drive with PMSM (permanent magnet synchronous motor) takes into account time varying load torque and motor copper losses.

Generally, we consider the system of linear differential equations

$$
\begin{aligned}
& y_{1}^{\prime}(t)=y_{2}(t)+f_{1}(t) \\
& a_{1} y_{2}{ }^{\prime}(t)=y_{3}(t)-y_{4}(t)+f_{2}(t) \\
& y_{3}^{\prime}(t)=a_{2} y_{2}(t)-a_{3} y_{3}(t)+f_{3}(t) \\
& y_{4}^{\prime}(t)=f_{4}(t) \\
& \quad \text { The DTM brings the resulting system } \\
& Y_{1}(n+1)=Y_{2}(n)+F_{1}(n) \\
& a_{1} Y_{2}(n+1)=Y_{3}(n)-Y_{4}(n)+F_{2}(n) \\
& Y_{3}(n+1)=a_{2} Y_{2}(n)+a_{3} Y_{3}(n)+F_{3}(n) \\
& Y_{4}(n+1)=F_{4}(n)
\end{aligned}
$$

The system (26) with $f_{i}(t)=0, i=1,2, f_{3}(t)=0.39$ and $f_{4}(t)=a_{4} t+a_{5}$ is studied in [6]. Parameters for this model of an engine are given in [8]. The numerical solution by Matlab computed for initial conditions $y_{1}(0)=y_{2}(0)=y_{3}(0)=y_{4}(0)=0$ and parameters $a_{1}=0.05, a_{2}=-\frac{1}{1.3}, a_{3}=0.39^{2}, a_{4}=a_{5}=1$ is

$y_{1}(t)=3.9 t^{2}-3.333 t^{3}-5.833 t^{4}+2.412 t^{5}+\ldots$

$y_{2}(t)=7.8 t-10 t^{2}-23.33 t^{3}+12.06 t^{4}+18.315 t^{5}+\ldots$ $y_{3}(t)=0.39 t-3 t^{2}+2.412 t^{3}+4.578 t^{4}-1.716 t^{5}+\ldots$ $y_{4}(t)=t+0.5 t^{2}$

\section{Conclusion}

We used the differential transformation method DTM for two different problems - coupled nonlinear oscillators and energy optimal speed and position control of PMSM drive. Solutions to linear and nonlinear systems of ODE are calculated for systems with power and exponential types of RHS.

\section{Acknowledgements}

The first author gratefully acknowledges the Scientific Grant Agency (VEGA) of the Ministry of Education of the Slovak Republic and the Slovak Academy of Sciences for supporting this work under Grant No. 1/1245/12. 


\section{References}

[1] ARONSON, D. G, ERMENTROUT, G. B., KOPELL, N.: Amplitude Response of Coupled Oscillators, Physica D, 41, 1990, pp. 403449.

[2] MIRZAEE, F.: Differential Transform Method for Solving Linear and Nonlinear Systems of Ordinary Differential Equations, Applied Mathematical Sciences, vol. 5, 2011, No. 70, pp. 3465-3472.

[3] KHAN, Y., SVOBODA, Z., SMARDA, Z.: Solving Certain Classes of Lane-n Type Equations Using the Differential Transformation Method, Advances in Difference Equations, 2012, 174.

[4] REBENDA, J., SMARDA, Z.: Stability and Asymptotic Properties of a System of Functional Differential Equations with Nonconstant Delays, Applied Mathematics and Computation 219, 2013, pp. 6622-6632.

[5] MARSDEN, J. E., MCCRACKEN, M.: The Hopf Bifurcation and Its Applications, Springer-Verlag, Applied Mathematical Sciences, New York, 1976.

[6] VITEK, J., FTOREK, B., BUTKO, P., FEDOR, T.: Energy Optimal Control of PMSM Drive for Time-varying Load Torque, Applied Mechanics and Materials, vol. 710, 2015, pp. 67-75.

[7] FTOREK, B., ORSANSKY, P.: Some Analytic Solutions of a Time-delay Differential System, Proc. of Interaction and feedbacks 2012: XIX-th national seminar, Prague: Institute of Thermomechanics, AS CR, 2012, pp. 5-10.

[8] VITEK, J., FTOREK, B.: Energy Efficient Speed and Position Control of Electric Drives with PMSM, Communications - Scientific Letters of the University of Zilina, vol. 16, No. 1, 2014, pp. 64-71. 